\title{
Second-harmonic generation of the lowest-order antisymmetric Lamb wave at a closed parallel crack
}

$\operatorname{AUTHOR}(\mathrm{S})$ :

Ye, Tianming; Biwa, Shiro; Mori, Naoki

\section{CITATION:}

Ye, Tianming ... [et all]. Second-harmonic generation of the lowest-order antisymmetric Lamb wave at a closed parallel crack. Journal of the Acoustical Society of America 2020, 148(4): 2073-2085

\section{ISSUE DATE:}

2020-10

URL:

http://hdl.handle.net/2433/255598

\section{RIGHT:}

Copyright 2020 Acoustical Society of America. This article may be downloaded for personal use only. Any other use requires prior permission of the author and the Acoustical Society of America.; The full-text file will be made open to the public on 14 April 2021 in accordance with publisher's 'Terms and Conditions for Self-Archiving'. 


\section{Second-harmonic generation of the lowest-order antisymmetric Lamb wave at a closed parallel crack}

Tianming Ye, Shiro Biwa, and Naoki Mori

Citation: The Journal of the Acoustical Society of America 148, 2073 (2020); doi: 10.1121/10.0002171

View online: https://doi.org/10.1121/10.0002171

View Table of Contents: https://asa.scitation.org/toc/jas/148/4

Published by the Acoustical Society of America

\section{ARTICLES YOU MAY BE INTERESTED IN}

Numerical study of acoustic focusing using a bianisotropic acoustic lens

The Journal of the Acoustical Society of America 148, EL365 (2020); https://doi.org/10.1121/10.0002137

Blind estimation of the direct-to-reverberant ratio using a beta distribution fit to binaural coherence

The Journal of the Acoustical Society of America 148, EL359 (2020); https://doi.org/10.1121/10.0002144

Distribution of the two-point product of complex amplitudes in the fully saturated scattering regime

The Journal of the Acoustical Society of America 148, EL347 (2020); https://doi.org/10.1121/10.0002143

Acoustic source localization with the angular spectrum approach in continuously stratified media The Journal of the Acoustical Society of America 148, EL333 (2020); https://doi.org/10.1121/10.0002095

A computational method whose time had come

The Journal of the Acoustical Society of America 148, R7 (2020); https://doi.org/10.1121/10.0002055

Multi-resolution estimation of the interference spectrum per pair of modes in the frequency domain

The Journal of the Acoustical Society of America 148, EL340 (2020); https://doi.org/10.1121/10.0002136

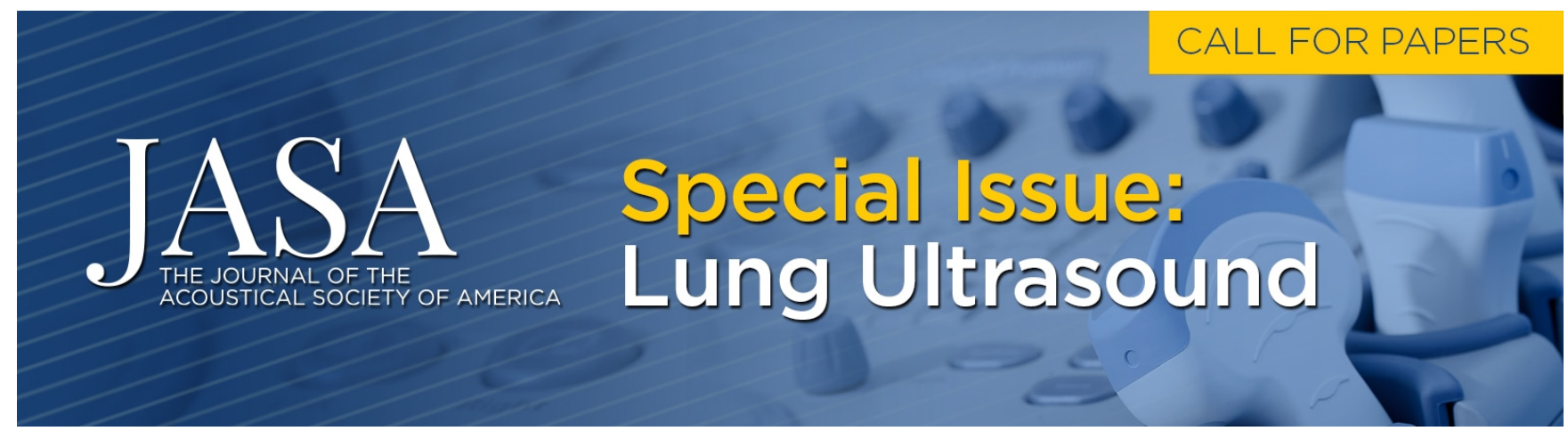




\title{
Second-harmonic generation of the lowest-order antisymmetric Lamb wave at a closed parallel crack
}

\author{
Tianming Ye, ${ }^{1}$ Shiro Biwa, ${ }^{2, a)}$ and Naoki Mori ${ }^{2}$ \\ ${ }^{1}$ Institute of Acoustics, Tongji University, No. 1239 Siping Road, Shanghai 200092, China \\ ${ }^{2}$ Department of Aeronautics and Astronautics, Graduate School of Engineering, Kyoto University, Katsura, Nishikyo-ku, \\ Kyoto 615-8540, Japan
}

\begin{abstract}
:
The second-harmonic generation of the fundamental antisymmetric Lamb wave at a closed parallel crack in an elastic plate is studied by numerical analysis. The closed crack is modeled as a spring-type interface with quadratic nonlinearity. Based on a perturbation method, the problem of nonlinear Lamb wave scattering is decomposed into two linearized problems, i.e., for the linear reflection/transmission of the incident Lamb wave at the crack and for the generation/radiation of the second-harmonic Lamb waves due to the contact nonlinearity. The reduced problems are solved by the finite element method in the frequency domain. Numerical results demonstrate significant effects of the crack resonance on the linear and nonlinear Lamb wave scattering responses, which appear as sharp peaks/dips in the reflection/transmission spectra and enhanced second-harmonic amplitudes at some frequencies. Two simple frequency selection rules are established which explain the enhanced generation of the second-harmonic Lamb waves. The time-domain analysis is also carried out to supplement the frequency-domain analysis, which confirms that the incident Lamb wave interacts with the crack at some specific frequencies in its bandwidth in a selective manner and enhances the generation of the second-harmonic components. (C) 2020 Acoustical Society of America. https://doi.org/10.1121/10.0002171
\end{abstract}

(Received 23 May 2020; revised 19 September 2020; accepted 23 September 2020; published online 14 October 2020)

[Editor: Nicole Kessissoglou]

Pages: 2073-2085

\section{INTRODUCTION}

Elastic waves propagate as guided modes in thin-walled structures such as plates and shells. Guided waves in plates are known as Lamb waves and have long been applied to nondestructive testing of plate-like structures. ${ }^{1,2}$ The scattering characteristics of Lamb waves by different types of defects in plates have been studied by a number of investigators. $^{3-11}$ Among others, Rokhlin ${ }^{3,4}$ analyzed the Lamb wave scattering by a finite crack parallel to the surfaces of an elastic plate and the associated resonant behavior due to the multiple reflection at two ends of the crack. The Lamb wave scattering characteristics of perpendicular cracks as well as notches were analyzed by Castaings et al., ${ }^{7}$ Lowe and Diligent, ${ }^{8}$ Lowe et al., ${ }^{9}$ and Flores-López and Gregory. ${ }^{11}$

In actual structures, crack-type defects can be closed with their faces in mutual contact due to external loads or temperature variations. Closed cracks show different interaction characteristics with Lamb waves from those of open cracks and can be more difficult to detect or characterize. ${ }^{12-15}$ For example, Kundu et al. ${ }^{12}$ have shown both theoretically and experimentally that the phase velocities of most Lamb modes are insensitive to the presence of a tightly closed interface in a plate except for a certain antisymmetric mode (A1 mode). Other studies ${ }^{13-15}$ have shown that the closure of a crack tends

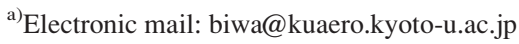

to reduce its wave-scattering magnitude and makes it more difficult to detect by Lamb waves.

While closed cracks can be difficult to characterize using conventional ultrasonic methods, they are known to generate the spectral components that differ from the incident wave frequency, such as higher harmonics and mixed frequency components, due to the nonlinear behavior of contacting crack surfaces, the so-called contact acoustic nonlinearity. ${ }^{16}$ Nonlinear features of the Lamb wave interaction with closed cracks are thus expected to provide a promising supplementary means to detect them in a sensitive manner. Recently, Yelve et al. ${ }^{17-19}$ studied the feasibility of Lamb-wave based nonlinear ultrasonic techniques for detection and localization of closed delamination-type defects in bonded metallic plates as well as composite laminates. Higher harmonic generation characteristics of Lamb waves at closed delaminations in composite laminates were also investigated by other investigators. ${ }^{20,21}$

In order to exploit the applicability of Lamb-wavebased nonlinear ultrasonic techniques for closed cracks, a number of theoretical and numerical studies have also been carried out. Shkerdin and Glorieux ${ }^{22,23}$ analyzed the nonlinear interaction of a low-frequency pumping Lamb wave and a high-frequency probing Lamb wave at a contacting delamination in bilayer structures. Delrue and Van Den Abeele $^{24,25}$ and Singh et al. ${ }^{26}$ analyzed the higher harmonic and subharmonic generation at a closed delamination in composite laminates using the finite element method. 
In these studies, the cracks (delaminations) are modeled as clapping surfaces that open during the tensile phase of the wave and hit each other and get in contact during the compressive phase. On the other hand, tightly closed cracks may remain in contact even in the tensile phase, but the stiffness of their contacting surfaces varies depending on the instantaneous pressure acting on them. Such behavior can be simulated by using nonlinear spring-type interface models ${ }^{27-29}$ which give the interfacial stresses as nonlinear functions of the relative displacements of both surfaces. Foregoing works have shown that nonlinear spring-type interface models are capable of describing the linear (reflection and transmission) as well as nonlinear (harmonic generation) characteristics of such contacting interfaces subjected to bulk ultrasonic waves. ${ }^{30-32}$ For Lamb waves, the reflection and transmission at an imperfect joint of plates ${ }^{33-35}$ and a closed crack in a plate $^{15}$ have been studied based on linear spring-type interface models. Recently, Mori et al. ${ }^{36}$ analyzed the secondharmonic generation of the fundamental symmetric Lamb wave at a nonlinear spring-type joint between two plates both in frequency and time domains. For closed cracks in plates, however, it still remains as an intriguing issue to analyze the nonlinear behavior of Lamb waves based on such models.

In this paper, the scattering and the second-harmonic generation of the fundamental antisymmetric Lamb wave at a closed parallel crack in an elastic plate is investigated by numerical analysis based on a nonlinear spring-type interface model. Although this model has been proven to reproduce the second-harmonic generation at contacting interfaces very well, its application to the analysis of nonlinear Lamb wave scattering by a closed crack is a new feature addressed in the present study. The analysis can be facilitated by assuming weak nonlinearity and using a perturbation method, which enables us to decompose the analysis into two parts for the linear and the second-harmonic responses. A preliminary analysis of this problem has been reported by Biwa and Ito, ${ }^{37}$ but more detailed formulations and numerical results are presented in this paper. The corresponding problem for an open parallel crack, hence without nonlinear effects, was analyzed earlier by Rokhlin ${ }^{3,4}$ who showed a significant effect of crack resonance on the Lamb wave scattering characteristics. The present study incorporates a closed crack and the associated nonlinear effect in the Lamb wave scattering analysis based on the nonlinear spring-type interface model, and the role of crack resonance in the second-harmonic generation of Lamb waves is discussed. Examination of this issue is of great interest as the enhancement of contact acoustic nonlinearity by the defect resonance is attracting much attention in current nonlinear ultrasonic nondestructive evaluation techniques. ${ }^{26,38,39}$

This paper is structured as follows. In Sec. II, the scattering problem of the fundamental antisymmetric Lamb wave by a closed parallel crack in an elastic plate is formulated by modeling the crack as a nonlinear spring-type interface. Based on the perturbation analysis assuming weak nonlinearity of the crack, the nonlinear problem is decomposed into two linear problems describing the linear scattering response and the perturbation corresponding to the nonlinear response, respectively. In Sec. III, these problems are described in the frequency domain, and the finite element method is employed to solve them. In the numerical analysis, the spring-type interface is replaced by a thin elastic layer with equivalent elastic constants, for which the validity is discussed in the Appendix. In Sec. IV, the numerical results of the linear as well as second-harmonic responses of the closed crack to the Lamb wave are presented, and the effects of the incident-wave frequency as well as the crack length are discussed. In Sec. V, the analysis is supplemented by the time-domain analysis and the spectral components arising from the nonlinear Lamb wave scattering are discussed. In Sec. VI, the summary of the present analysis is provided.

\section{FORMULATION OF THE PROBLEM}

Consider an infinitely extended thin isotropic and linear elastic plate (density $\rho$, longitudinal wave velocity $c_{\mathrm{L}}$, shear wave velocity $c_{\mathrm{T}}$ ) of thickness $d$, which has a parallel straight crack of length $a$ lying at the distance $b$ from the lower surface as shown in Fig. 1 . The $x_{1}-x_{2}$ coordinate system is set with the mid-plane of the plate at $x_{2}=0$ and the normal to the plate surfaces in the $x_{2}$-axis. The crack lies at $x_{2}=g=-d / 2+b$ and is centered at $x_{1}=0$, extending over $-a / 2<x_{1}<a / 2$. The crack is assumed to be closed with its surfaces being in contact with each other. In the present study, the closed crack is modeled as a nonlinear springtype interface where the stress components are assumed continuous, but the displacements can have finite jumps. Specifically, the normal and shear stresses, $\sigma_{22}$ and $\sigma_{12}$, respectively, on the crack surfaces are given by quadratic functions of the relative displacements therein, i.e.,

$$
\begin{aligned}
\sigma_{22}\left(x_{1}, g^{+}, t\right)= & \sigma_{22}\left(x_{1}, g^{-}, t\right) \\
= & K_{\mathrm{N}}\left[y_{2}\left(x_{1}, t\right)-\beta y_{2}\left(x_{1}, t\right)^{2}-\gamma y_{1}\left(x_{1}, t\right)^{2}\right], \\
& -\frac{a}{2}<x_{1}<\frac{a}{2}, \\
\sigma_{12}\left(x_{1}, g^{+}, t\right)= & \sigma_{12}\left(x_{1}, g^{-}, t\right)=K_{\mathrm{T}}\left[y_{1}\left(x_{1}, t\right)\right. \\
& \left.-\chi y_{1}\left(x_{1}, t\right) y_{2}\left(x_{1}, t\right)\right], \quad-\frac{a}{2}<x_{1}<\frac{a}{2},
\end{aligned}
$$

where $K_{\mathrm{N}}$ and $K_{\mathrm{T}}$ are the normal and tangential interfacial stiffnesses, respectively, and $\beta, \gamma$ and $\chi$ are the parameters representing the nonlinearity of the crack surfaces. ${ }^{32}$ The relative displacements of the crack surfaces are defined by

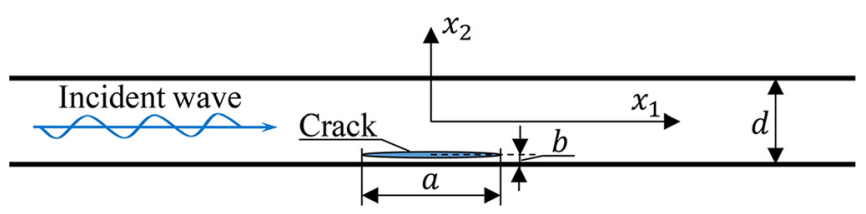

FIG. 1. (Color online) An elastic plate with a parallel crack. 


$$
\begin{gathered}
y_{1}\left(x_{1}, t\right)=u_{1}\left(x_{1}, g^{+}, t\right)-u_{1}\left(x_{1}, g^{-}, t\right), \\
y_{2}\left(x_{1}, t\right)=u_{2}\left(x_{1}, g^{+}, t\right)-u_{2}\left(x_{1}, g^{-}, t\right),
\end{gathered}
$$

where $u_{1}$ and $u_{2}$ are the horizontal and vertical displacement components. This model can be interpreted as the Taylor expansion of the interfacial stresses with respect to the relative displacements, taken up to the second-order terms and restricted to the symmetric response with respect to the shear direction (for more details, see Ref. 32). It can also be obtained as a limiting case of a thin nonlinear elastic interphase layer, as discussed by An et al. ${ }^{40}$ and Zhang et al. ${ }^{41} \mathrm{It}$ has been used in the analysis of the second-harmonic generation at contacting interfaces for normally incident waves $^{28,30}$ and for more general situations. ${ }^{36,42}$ Similar models can be found in other works ${ }^{27,29,31}$ on the contact acoustic nonlinearity. For the sake of brevity, the nonlinear effects of the closed crack are solely represented by the term $\beta y_{2}\left(x_{1}, t\right)^{2}$ on the right-hand side of Eq. (1) in the present analysis, so the parameters $\gamma$ and $\chi$ are set as zero hereafter. The upper and lower surfaces of the plate are assumed to be traction-free, i.e.,

$$
\sigma_{22}\left(x_{1}, \pm \frac{d}{2}, t\right)=\sigma_{12}\left(x_{1}, \pm \frac{d}{2}, t\right)=0
$$

The plate is subjected to the incidence of the fundamental antisymmetric Lamb wave (A0 mode) with the frequency $f_{0}$ (the angular frequency $\omega_{0}=2 \pi f_{0}$ ) propagating in the positive $x_{1}$-direction, which has the following displacement components in a homogeneous plate:

$$
u_{\alpha}^{\mathrm{Inc}}\left(x_{1}, x_{2}, t\right)=\operatorname{Re}\left[U_{\alpha}^{\mathrm{A} 0}\left(x_{2}\right) \exp \left[\mathrm{i}\left\{k^{\mathrm{A} 0}\left(\omega_{0}\right) x_{1}-\omega_{0} t\right\}\right]\right],
$$

where $k^{\mathrm{A} 0}\left(\omega_{0}\right)$ is the wavenumber of the $\mathrm{A} 0$ mode at the angular frequency $\omega_{0}$ and $U_{\alpha}^{\mathrm{A} 0}\left(x_{2}\right)$ denotes the throughthickness displacement profile of that mode, which can be found in ordinary textbooks. ${ }^{43}$ The incidence of the A0 mode is studied here since its displacement is dominated by the vertical component, which is perpendicular to the crack surfaces and expected to cause significant nonlinear response. The incident $\mathrm{A} 0 \mathrm{Lamb}$ wave is scattered by the crack. This phenomenon is governed by the following twodimensional, plane-strain Navier equation:

$$
\left(c_{\mathrm{L}}^{2}-c_{\mathrm{T}}^{2}\right) \frac{\partial^{2} u_{\gamma}}{\partial x_{\alpha} \partial x_{\gamma}}+c_{\mathrm{T}}^{2} \frac{\partial^{2} u_{\alpha}}{\partial x_{\gamma} \partial x_{\gamma}}=\frac{\partial^{2} u_{\alpha}}{\partial t^{2}}, \quad \alpha=1,2 .
$$

Here and hereafter, the two-dimensional summation convention is adopted, i.e., an index repeated twice in a term implies the sum of that term with this index for 1 and 2 .

The problem to be analyzed thus consists of Eq. (6) with the boundary conditions of Eqs. (1)-(4) for the incident wave of Eq. (5). Due to the nonlinearity of the crack surfaces, it is not possible to obtain an exact solution of the problem analytically. In the present analysis, the nonlinear effect of the closed crack is assumed sufficiently weak, and a perturbation method is used to obtain an approximate solution. ${ }^{32}$ Namely, the solution $u_{\alpha}\left(x_{1}, x_{2}, t\right)$ is decomposed into two parts as

$$
u_{\alpha}\left(x_{1}, x_{2}, t\right)=\bar{u}_{\alpha}\left(x_{1}, x_{2}, t\right)+\hat{u}_{\alpha}\left(x_{1}, x_{2}, t\right),
$$

where $\bar{u}_{\alpha}$ represents the linear response obtained by neglecting the nonlinear term in the boundary conditions of the crack and $\hat{u}_{\alpha}$ represents the small perturbation from the linear response. The other associated quantities (relative displacements and stresses) are likewise defined with a superposed bar for the linear response and with a hat for the perturbation.

The linear response is governed by Eqs. (5) and (6) with $\bar{u}_{\alpha}$ in place of $u_{\alpha}$, together with Eq. (4) with $\bar{\sigma}_{\alpha \gamma}$ in place of $\sigma_{\alpha \gamma}$, and

$$
\begin{gathered}
\bar{\sigma}_{22}\left(x_{1}, g^{+}, t\right)=\bar{\sigma}_{22}\left(x_{1}, g^{-}, t\right)=K_{\mathrm{N}} \bar{y}_{2}\left(x_{1}, t\right), \\
-\frac{a}{2}<x_{1}<\frac{a}{2} \\
\bar{\sigma}_{12}\left(x_{1}, g^{+}, t\right)=\bar{\sigma}_{12}\left(x_{1}, g^{-}, t\right)=K_{\mathrm{T}} \bar{y}_{1}\left(x_{1}, t\right), \\
-\frac{a}{2}<x_{1}<\frac{a}{2}, \\
\bar{y}_{1}\left(x_{1}, t\right)=\bar{u}_{1}\left(x_{1}, g^{+}, t\right)-\bar{u}_{1}\left(x_{1}, g^{-}, t\right), \\
\bar{y}_{2}\left(x_{1}, t\right)=\bar{u}_{2}\left(x_{1}, g^{+}, t\right)-\bar{u}_{2}\left(x_{1}, g^{-}, t\right),
\end{gathered}
$$

as the boundary conditions at the crack. It is convenient to introduce the complex-value expression

$$
\begin{aligned}
& \bar{u}_{\alpha}\left(x_{1}, x_{2}, t\right)=\operatorname{Re}\left[\bar{U}_{\alpha}\left(x_{1}, x_{2}\right) \exp \left(-\mathrm{i} \omega_{0} t\right)\right], \\
& \bar{y}_{\alpha}\left(x_{1}, x_{2}, t\right)=\operatorname{Re}\left[\bar{Y}_{\alpha}\left(x_{1}, x_{2}\right) \exp \left(-\mathrm{i} \omega_{0} t\right)\right], \\
& \bar{\sigma}_{\alpha \beta}\left(x_{1}, x_{2}, t\right)=\operatorname{Re}\left[\bar{\Sigma}_{\alpha \beta}\left(x_{1}, x_{2}\right) \exp \left(-\mathrm{i} \omega_{0} t\right)\right],
\end{aligned}
$$

and formulate the problem in the frequency domain as

$$
\begin{gathered}
\left(c_{\mathrm{L}}^{2}-c_{\mathrm{T}}^{2}\right) \frac{\partial^{2} \bar{U}_{\gamma}}{\partial x_{\alpha} \partial x_{\gamma}}+c_{\mathrm{T}}^{2} \frac{\partial^{2} \bar{U}_{\alpha}}{\partial x_{\gamma} \partial x_{\gamma}}+\omega_{0}^{2} \bar{U}_{\alpha}=0, \\
\bar{\Sigma}_{22}\left(x_{1}, \pm \frac{d}{2}\right)=\bar{\Sigma}_{12}\left(x_{1}, \pm \frac{d}{2}\right)=0, \\
\bar{\Sigma}_{22}\left(x_{1}, g^{+}\right)=\bar{\Sigma}_{22}\left(x_{1}, g^{-}\right)=K_{\mathrm{N}} \bar{Y}_{2}\left(x_{1}\right), \\
-\frac{a}{2}<x_{1}<\frac{a}{2} \\
\bar{\Sigma}_{12}\left(x_{1}, g^{+}\right)=\bar{\Sigma}_{12}\left(x_{1}, g^{-}\right)=K_{\mathrm{T}} \bar{Y}_{1}\left(x_{1}\right), \\
-\frac{a}{2}<x_{1}<\frac{a}{2}
\end{gathered}
$$

for the incident wave given by

$$
\bar{U}_{\alpha}^{\mathrm{Inc}}\left(x_{1}, x_{2}\right)=U_{\alpha}^{\mathrm{A} 0}\left(x_{2}\right) \exp \left[\mathrm{i} k^{\mathrm{A} 0}\left(\omega_{0}\right) x_{1}\right] .
$$

The linear response describes the scattering of the incident A0-mode Lamb wave by a closed crack modeled as a linear spring-type interface. After the linear response is 


$$
\begin{gathered}
\hat{\sigma}_{12}\left(x_{1}, g^{+}, t\right)=\hat{\sigma}_{12}\left(x_{1}, g^{-}, t\right)=K_{\mathrm{T}} \hat{y}_{1}\left(x_{1}, t\right), \\
-\frac{a}{2}<x_{1}<\frac{a}{2}, \\
\hat{y}_{1}\left(x_{1}, t\right)=\hat{u}_{1}\left(x_{1}, g^{+}, t\right)-\hat{u}_{1}\left(x_{1}, g^{-}, t\right), \\
\hat{y}_{2}\left(x_{1}, t\right)=\hat{u}_{2}\left(x_{1}, g^{+}, t\right)-\hat{u}_{2}\left(x_{1}, g^{-}, t\right) .
\end{gathered}
$$

$$
\begin{aligned}
& \tilde{\Sigma}_{22}\left(x_{1}, \pm \frac{d}{2}\right)=\tilde{\Sigma}_{12}\left(x_{1}, \pm \frac{d}{2}\right)=0, \\
& \tilde{\Sigma}_{22}\left(x_{1}, g^{+}\right)=\tilde{\Sigma}_{22}\left(x_{1}, g^{-}\right)=K_{\mathrm{N}}\left[\tilde{Y}_{2}\left(x_{1}\right)-\beta F\left(x_{1}\right)\right], \\
& -\frac{a}{2}<x_{1}<\frac{a}{2}, \\
& \tilde{\Sigma}_{12}\left(x_{1}, g^{+}\right)=\tilde{\Sigma}_{12}\left(x_{1}, g^{-}\right)=K_{\mathrm{T}} \tilde{Y}_{1}\left(x_{1}\right), \\
& -\frac{a}{2}<x_{1}<\frac{a}{2},
\end{aligned}
$$

where

$$
\begin{aligned}
F\left(x_{1}\right)= & \frac{1}{2}\left\{\left(\operatorname{Re}\left[\bar{Y}_{2}\left(x_{1}\right)\right]\right)^{2}+\left(\operatorname{Im}\left[\bar{Y}_{2}\left(x_{1}\right)\right]\right)^{2}\right\} \\
& \times \exp \left[-\mathrm{i} \phi\left(x_{1}\right)\right] .
\end{aligned}
$$

The above problem contains $\bar{y}_{2}\left(x_{1}, t\right)^{2}$ on the right-hand side of Eq. (19) from the linear solution, which can be calculated as

$$
\begin{aligned}
\bar{y}_{2}\left(x_{1}, t\right)^{2}= & \left(\operatorname{Re}\left[\bar{Y}_{2}\left(x_{1}\right) \exp \left(-\mathrm{i} \omega_{0} t\right)\right]\right)^{2} \\
= & \frac{1}{2}\left\{\left(\operatorname{Re}\left[\bar{Y}_{2}\left(x_{1}\right)\right]\right)^{2}+\left(\operatorname{Im}\left[\bar{Y}_{2}\left(x_{1}\right)\right]\right)^{2}\right\} \\
& +\frac{1}{2}\left\{\left(\operatorname{Re}\left[\bar{Y}_{2}\left(x_{1}\right)\right]\right)^{2}+\left(\operatorname{Im}\left[\bar{Y}_{2}\left(x_{1}\right)\right]\right)^{2}\right\} \\
& \times \cos \left(2 \omega_{0} t+\phi\left(x_{1}\right)\right),
\end{aligned}
$$

where the variable $\phi\left(x_{1}\right)$ is defined by

$$
\begin{aligned}
& \cos \phi\left(x_{1}\right)=\frac{\left(\operatorname{Re}\left[\bar{Y}_{2}\left(x_{1}\right)\right]\right)^{2}-\left(\operatorname{Im}\left[\bar{Y}_{2}\left(x_{1}\right)\right]\right)^{2}}{\left(\operatorname{Re}\left[\bar{Y}_{2}\left(x_{1}\right)\right]\right)^{2}+\left(\operatorname{Im}\left[\bar{Y}_{2}\left(x_{1}\right)\right]\right)^{2}}, \\
& \sin \phi\left(x_{1}\right)=-\frac{2 \operatorname{Re}\left[\bar{Y}_{2}\left(x_{1}\right)\right] \operatorname{Im}\left[\bar{Y}_{2}\left(x_{1}\right)\right]}{\left(\operatorname{Re}\left[\bar{Y}_{2}\left(x_{1}\right)\right]\right)^{2}+\left(\operatorname{Im}\left[\bar{Y}_{2}\left(x_{1}\right)\right]\right)^{2}} .
\end{aligned}
$$

The term $K_{\mathrm{N}} \beta \bar{y}_{2}\left(x_{1}, t\right)^{2}$ on the right-hand side of Eq. (19) is a source to generate the perturbed wave field, which is given by the superposition of the static $(\omega=0)$ component $u_{\alpha}^{0}\left(x_{1}, x_{2}, t\right)$ and the second-harmonic $\left(\omega=2 \omega_{0}\right)$ component $\tilde{u}_{\alpha}\left(x_{1}, x_{2}, t\right)$, i.e., $\hat{u}_{\alpha}=u_{\alpha}^{0}+\tilde{u}_{\alpha}$. Our attention in this study is focused on the second-harmonic generation. The secondharmonic part can also be obtained in the frequency domain, i.e.,

$$
\begin{aligned}
& \tilde{u}_{\alpha}\left(x_{1}, x_{2}, t\right)=\operatorname{Re}\left[\tilde{U}_{\alpha}\left(x_{1}, x_{2}\right) \exp \left(-2 \mathrm{i} \omega_{0} t\right)\right], \\
& \tilde{y}_{\alpha}\left(x_{1}, x_{2}, t\right)=\operatorname{Re}\left[\tilde{Y}_{\alpha}\left(x_{1}, x_{2}\right) \exp \left(-2 \mathrm{i} \omega_{0} t\right)\right], \\
& \tilde{\sigma}_{\alpha \beta}\left(x_{1}, x_{2}, t\right)=\operatorname{Re}\left[\tilde{\Sigma}_{\alpha \beta}\left(x_{1}, x_{2}\right) \exp \left(-2 \mathrm{i} \omega_{0} t\right)\right],
\end{aligned}
$$

by solving the problem

$$
\left(c_{\mathrm{L}}^{2}-c_{\mathrm{T}}^{2}\right) \frac{\partial^{2} \tilde{U}_{\gamma}}{\partial x_{\alpha} \partial x_{\gamma}}+c_{\mathrm{T}}^{2} \frac{\partial^{2} \tilde{U}_{\alpha}}{\partial x_{\gamma} \partial x_{\gamma}}+\left(2 \omega_{0}\right)^{2} \tilde{U}_{\alpha}=0,
$$

\section{NUMERICAL ANALYSIS}

\section{A. Finite element modeling}

Based on the formulation given above, the scattering of the A0-mode Lamb wave by a closed parallel crack is now analyzed for a low-frequency range below the cut-off frequency of the A1 mode, $f_{\mathrm{A} 1}=c_{\mathrm{T}} /(2 d)$. Specifically, the linear response is analyzed for the normalized range of the incident-wave frequency $0<f_{0} d / c_{\mathrm{T}}<0.2$ to elucidate the linear scattering behavior of the A0-mode Lamb wave by the crack. Then, the second-harmonic response is analyzed for the normalized incident-wave frequency $0<f_{0} d / c_{\mathrm{T}}$ $<0.1$, which corresponds to the frequency range of the second-harmonics of $0<2 f_{0} d / c_{\mathrm{T}}<0.2$. In both analyses, the A 0 and S0 modes are the only propagating Lamb modes in the homogeneous plate. Based on the perturbation analysis introduced in Sec. II, the linear and the second-harmonic responses are analyzed by utilizing the commercial finite element analysis software Comsol Multiphysics Ver. 5.4. All the computations are carried out in the frequency domain within the two-dimensional plane-strain framework. The schematic of the computational model used for the analysis is shown in Fig. 2. In Fig. 2, the region of the plate of length $120 d$ is divided into finite elements and connected to the perfectly matched layers (PMLs) of length $40 \mathrm{~d}$ on both sides to simulate the infinitely extended plate. Quadrilateral elements are used for mesh division with the maximum element size $d / 20$, which ensures that the elements are smaller than $1 / 100$ of the shear wave length in the frequency range $0<f_{0} d / c_{\mathrm{T}}<0.2$. The crack length is varied as $a=2 d, 4 d$, and $6 d$ to examine its influence on the linear and nonlinear scattering behavior, while the crack location (distance from the lower surface of the plate) is fixed as $b=0.1 d$. In the present analysis, the material constants of aluminum alloy are used for the plate $\left(\rho=2700 \mathrm{~kg} / \mathrm{m}^{3}, c_{\mathrm{L}}=6400 \mathrm{~m} / \mathrm{s}, c_{\mathrm{T}}\right.$ $=3170 \mathrm{~m} / \mathrm{s})$. The value of $d$ is set as unity $(d=1 \mathrm{~mm})$ in the actual computations, but the results will be shown in the normalized form so that they are valid for other dimensions of the plate if the normalized variables are the same. In the analysis for the linear response, a pair of the point forces 


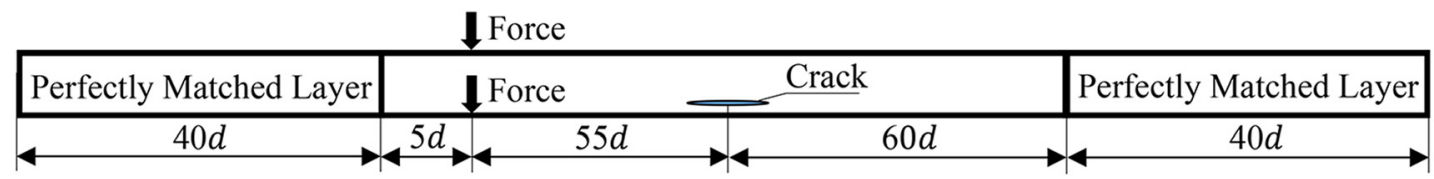

FIG. 2. (Color online) The numerical model of the plate with a crack.

with the same magnitude is applied in the vertical direction far away from the crack, at $x_{1}=-55 d$. This is effective to simulate the incidence of the A0-mode Lamb wave to the crack since the A0 mode is the only antisymmetric Lamb mode that can propagate in the plate in the assumed frequency range. The choice of the force magnitude is arbitrary, owing to the linearized nature of the problem.

The interfacial stiffnesses $K_{\mathrm{N}}$ and $K_{\mathrm{T}}$ represent the degree of crack closure. For contacting interfaces of solid bodies, the ratio of these stiffnesses $K_{\mathrm{T}} / K_{\mathrm{N}}$ is known to take different values, varying roughly between 0.2 and 0.6 depending on the material, surface topography, and applied pressure. ${ }^{44-48}$ In the present analysis, the fixed value of $K_{\mathrm{N}}$, i.e., $K_{\mathrm{N}} d /\left(\rho c_{\mathrm{T}}^{2}\right)=0.01$ and the fixed ratio of $K_{\mathrm{T}} / K_{\mathrm{N}}=0.4$ are used. Furthermore, the spring-type interface is replaced by a very thin elastic layer (thickness $\delta$ ) having zero mass density and elastic constants $\lambda_{\mathrm{C}}$ and $\mu_{\mathrm{C}}$, which are determined by the relations

$$
K_{\mathrm{N}}=\frac{\lambda_{\mathrm{C}}+2 \mu_{\mathrm{C}}}{\delta}, \quad K_{\mathrm{T}}=\frac{\mu_{\mathrm{C}}}{\delta}
$$

in accordance with the interfacial stiffnesses. ${ }^{49}$ The thickness of this layer is set as $\delta=10^{-6} d$. The validity of simulating the spring-type interface by a thin elastic layer is demonstrated in the Appendix by examining the dispersion relations of a bilayer plate structure with a spring-type interface and a three-layer plate with a thin elastic layer.

As explained above, the nonlinear Lamb wave scattering by the closed crack is analyzed in two steps in this study, first for the linear scattering and then for the secondharmonic generation. In each step, the problem is a linear one and solved in the frequency domain with the closed crack modeled as a linear spring-type interface. Furthermore, the spring-type interface is simulated by a thin elastic layer with equivalent elastic constants in the finite element analysis. It is, however, worth noting here that the nonlinear spring-type interface similar to Eqs. (1) and (2) has been directly modeled in the time domain by Zhang et ll. $^{41}$ using Comsol Multiphysics in their analysis of noncollinear mixing of bulk shear waves.

\section{B. Evaluation of linear response}

The finite element analysis for the linear response described by Eqs. (14)-(18) yields the displacement field with the incident-wave frequency in the plate. It gives the transmitted wave ahead of the crack $\left(x_{1} \gg 0\right)$, while it gives the superposition of the incident wave and the reflected wave behind the crack $\left(x_{1} \ll 0\right)$. In order to separate the reflected wave from the incident wave, a similar analysis is performed for the homogeneous plate without the crack, and the resulting wave field is assumed to be the incident wave field: the reflected wave field is then obtained by subtracting the incident wave field from the displacement field for the plate with the crack. In the frequency range analyzed here, the A0 and S0 modes are the only modes that propagate far away from the crack. At the position $x_{1}=50 \mathrm{~d}$ ahead of the crack and at $x_{1}=-50 \mathrm{~d}$ behind the crack, the displacements and stresses are decomposed into those of the A0 and S0 modes by

$$
\begin{aligned}
& \bar{U}_{1}^{\mathrm{A} 0}\left(x_{1}, x_{2}\right)=\frac{1}{2}\left\{\bar{U}_{1}\left(x_{1}, x_{2}\right)-\bar{U}_{1}\left(x_{1},-x_{2}\right)\right\}, \\
& \bar{U}_{2}^{\mathrm{A} 0}\left(x_{1}, x_{2}\right)=\frac{1}{2}\left\{\bar{U}_{2}\left(x_{1}, x_{2}\right)+\bar{U}_{2}\left(x_{1},-x_{2}\right)\right\}, \\
& \bar{U}_{1}^{\mathrm{S} 0}\left(x_{1}, x_{2}\right)=\frac{1}{2}\left\{\bar{U}_{1}\left(x_{1}, x_{2}\right)+\bar{U}_{1}\left(x_{1},-x_{2}\right)\right\}, \\
& \bar{U}_{2}^{\mathrm{S} 0}\left(x_{1}, x_{2}\right)=\frac{1}{2}\left\{\bar{U}_{2}\left(x_{1}, x_{2}\right)-\bar{U}_{2}\left(x_{1},-x_{2}\right)\right\}, \\
& \bar{\Sigma}_{11}^{\mathrm{A} 0}\left(x_{1}, x_{2}\right)=\frac{1}{2}\left\{\bar{\Sigma}_{11}\left(x_{1}, x_{2}\right)-\bar{\Sigma}_{11}\left(x_{1},-x_{2}\right)\right\}, \\
& \bar{\Sigma}_{12}^{\mathrm{A} 0}\left(x_{1}, x_{2}\right)=\frac{1}{2}\left\{\bar{\Sigma}_{12}\left(x_{1}, x_{2}\right)+\bar{\Sigma}_{12}\left(x_{1},-x_{2}\right)\right\}, \\
& \bar{\Sigma}_{11}^{\mathrm{S} 0}\left(x_{1}, x_{2}\right)=\frac{1}{2}\left\{\bar{\Sigma}_{11}\left(x_{1}, x_{2}\right)+\bar{\Sigma}_{11}\left(x_{1},-x_{2}\right)\right\}, \\
& \bar{\Sigma}_{12}^{\mathrm{S} 0}\left(x_{1}, x_{2}\right)=\frac{1}{2}\left\{\bar{\Sigma}_{12}\left(x_{1}, x_{2}\right)-\bar{\Sigma}_{12}\left(x_{1},-x_{2}\right)\right\} .
\end{aligned}
$$

In the complex-value representation, the energy flux across a cross-section of the plate is given by

$$
P=\frac{\mathrm{i} \omega}{4} \int_{-d / 2}^{d / 2}\left(\bar{U}_{1} \bar{\Sigma}_{11}^{*}+\bar{U}_{2} \bar{\Sigma}_{12}^{*}-\bar{U}_{1}^{*} \bar{\Sigma}_{11}-\bar{U}_{2}^{*} \bar{\Sigma}_{12}\right) \mathrm{d} x_{2}
$$

where * denotes a complex conjugate. Using the above expression, the energy flux is calculated for the reflected and transmitted $\mathrm{A} 0$ and $\mathrm{S} 0$ modes $\left(P_{\mathrm{RA} 0}, P_{\mathrm{RS} 0}, P_{\mathrm{TA} 0}, P_{\mathrm{TS} 0}\right)$ and normalized in the following way to obtain the reflection and transmission coefficients of two modes, i.e.,

$$
\begin{aligned}
& R_{\mathrm{A} 0}=\sqrt{\left|\frac{P_{\mathrm{RA} 0}}{P_{\mathrm{IA} 0}}\right|}, \quad R_{\mathrm{S} 0}=\sqrt{\left|\frac{P_{\mathrm{RS} 0}}{P_{\mathrm{IA} 0}}\right|}, \\
& T_{\mathrm{A} 0}=\sqrt{\left|\frac{P_{\mathrm{TA} 0}}{P_{\mathrm{IA} 0}}\right|}, \quad T_{\mathrm{S} 0}=\sqrt{\left|\frac{P_{\mathrm{TS} 0}}{P_{\mathrm{IA} 0}}\right|},
\end{aligned}
$$

where $P_{\text {IA0 }}$ represents the energy flux of the incident A0 mode. In this analysis, $P_{\mathrm{IA} 0}$ is determined for the 
homogeneous plate with no crack, at $x_{1}=0$ corresponding to the center of the crack.

\section{Evaluation of second-harmonic response}

The second-harmonic wave field is also solved by the finite element method in the absence of incident wave but with the wave source given by the quadratic nonlinearity of the defect as described by Eqs. (27)-(31). The relative displacements of the upper and lower surfaces of the crack (modeled as a thin layer) are extracted from the linearresponse analysis, and the normal stress $-K_{\mathrm{N}} \beta F\left(x_{1}\right)$ with $F\left(x_{1}\right)$ given in Eq. (31) is applied on the crack surfaces, i.e., on the upper and lower boundaries of the thin layer.

The second-harmonic response analysis gives the wave field with the double frequency $2 f_{0}$ radiated from the crack into both directions along the plate. Then, the displacements and stresses are evaluated at the same cross-sections as in the linear-response analysis, and the energy flux of the backscattered and forward-scattered second-harmonic A0 and S0 modes, denoted by $\tilde{P}_{\mathrm{RA} 0}, \tilde{P}_{\mathrm{RS} 0}, \tilde{P}_{\mathrm{TA} 0}, \tilde{P}_{\mathrm{TS} 0}$, respectively, are obtained at $x_{1}=-50 d$ and $x_{1}=50 d$ using Eq. (34) with the second-harmonic displacements $\tilde{U}_{\alpha}$ and stresses $\tilde{\Sigma}_{\alpha \gamma}$ for $\omega=2 \omega_{0}$. For the demonstration in Sec. IV, they are normalized as

$$
\begin{aligned}
& \tilde{R}_{\mathrm{A} 0}=\frac{1}{\beta A_{0}} \sqrt{\left|\frac{\tilde{P}_{\mathrm{RA} 0} \mid}{P_{\mathrm{IA} 0}}\right|}, \quad \tilde{R}_{\mathrm{S} 0}=\frac{1}{\beta A_{0}} \sqrt{\left|\frac{\tilde{P}_{\mathrm{RS} 0}}{P_{\mathrm{IA} 0}}\right|}, \\
& \tilde{T}_{\mathrm{A} 0}=\frac{1}{\beta A_{0}} \sqrt{\left|\frac{\tilde{P}_{\mathrm{TA} 0} \mid}{P_{\mathrm{IA} 0}}\right|}, \quad \tilde{T}_{\mathrm{S} 0}=\frac{1}{\beta A_{0}} \sqrt{\left|\frac{\tilde{P}_{\mathrm{TS} 0}}{P_{\mathrm{IA} 0}}\right|},
\end{aligned}
$$

where the reference displacement magnitude $A_{0}$ is chosen as the absolute value of the vertical displacement of the incident A0 mode at $x_{1}=0, x_{2}=0$ for each frequency.

\section{RESULTS AND DISCUSSION}

\section{A. Linear response}

The results for the plate with the crack length $a=4 d$ are first shown as the reference case. In Fig. 3, the reflection and transmission coefficients of the A0 and S0 modes are shown for the normalized incident-wave frequency range $0<f_{0} d / c_{\mathrm{T}}<0.2$. In Fig. 3, it is seen that the magnitude of the reflected $\mathrm{A} 0$ mode $\left(R_{\mathrm{A} 0}\right)$ has sharp peaks at several frequencies, accompanying sharp dips of the transmitted A0 mode $\left(T_{\mathrm{A} 0}\right)$ at almost the same frequencies. On the other hand, the magnitudes of reflected and transmitted S0 modes $\left(R_{\mathrm{S} 0}\right.$ and $\left.T_{\mathrm{S} 0}\right)$ remain negligibly small except at the frequencies where $R_{\mathrm{A} 0}$ shows sharp peaks. For the three lowest peak frequencies of $R_{\mathrm{A} 0}, f_{0} d / c_{\mathrm{T}}=0.0536,0.0574$, and 0.0754 , the distributions of the normal displacements of the crack, i.e., $\operatorname{Re}\left[U_{2}\right]$ on the upper and lower surfaces of the thin layer, are shown in Fig. 4, where the lower surface exhibits larger displacements since the sub-plate below the layer is relatively thin $(b=0.1 d)$. The corresponding problem for a plate with a traction-free crack located on the mid-

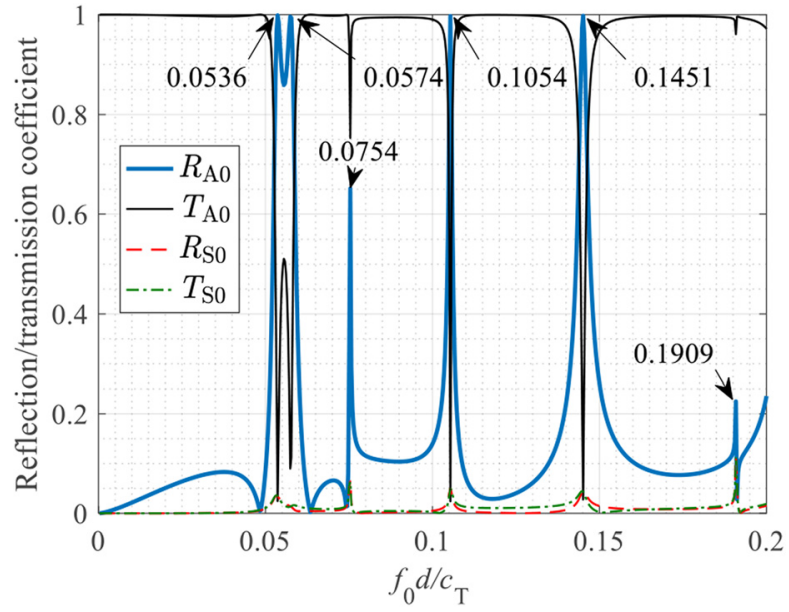

FIG. 3. (Color online) The reflection and transmission coefficients of the $\mathrm{A} 0$ and S0 modes against the normalized incident-wave frequency when $a$ $=4 d$.

plane has been analyzed by Rokhlin, ${ }^{3,4}$ who demonstrated sharp peaks and dips in the reflection/transmission responses due to the resonance at the crack. This interpretation is expected to be valid for the present case of the closed crack modeled as a spring-type interface. To confirm this, the eigenfrequency analysis is performed using Comsol Multiphysics for a segment of plate of the length $4 d$ containing the thin layer as shown in Fig. 5. The left and right boundaries of this segment are fixed while the upper and lower boundaries are traction-free. The displacement modes of the upper and lower surfaces of the thin layer are shown

(a)

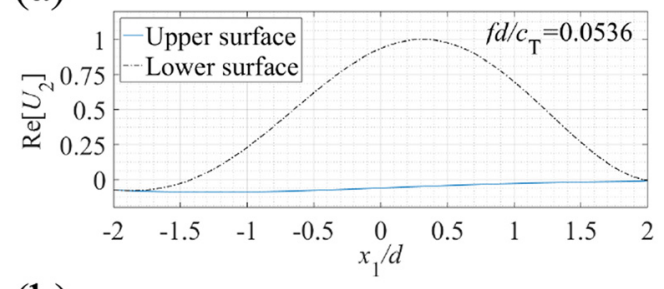

(b)

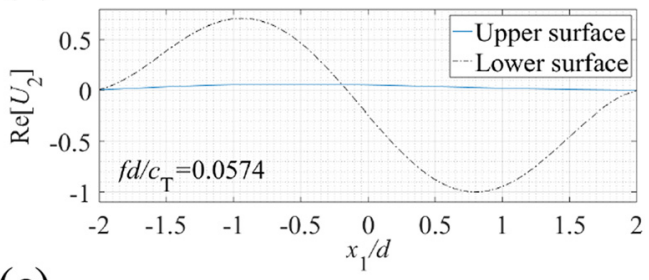

(c)

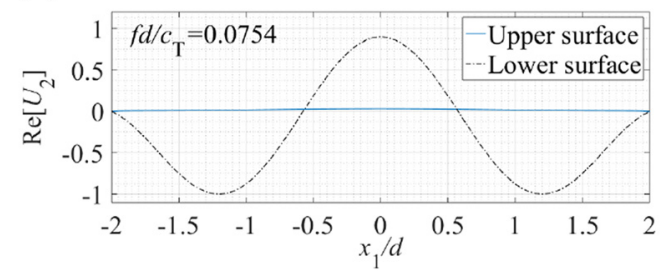

FIG. 4. (Color online) The displacement distribution of the upper and lower surfaces of the thin layer (closed crack) for $f_{0} d / c_{\mathrm{T}}=$ (a) 0.0536, (b) 0.0574, and (c) 0.0754 when $a=4 d$. 


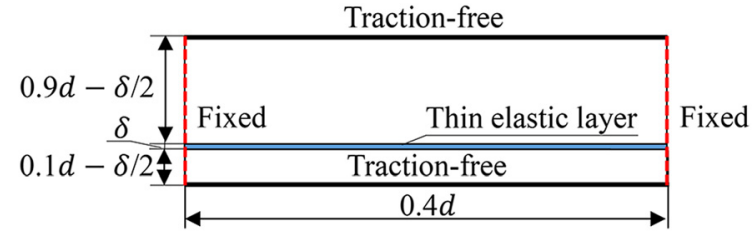

FIG. 5. (Color online) The finite plate model with a thin layer.

in Fig. 6 for the three lowest eigenfrequencies. The eigenfrequencies and the associated displacement profiles in Fig. 6 are in reasonable conformity with the peak frequencies and the displacement profiles in Fig. 4. The discrepancies between them should first come from the fact that the structure in Fig. 5 is fixed at both ends and each mode corresponds to free vibration, while the crack (thin layer) in our original problem of Fig. 2 is subjected to the incoming wave and radiates the scattered waves. Since it is embedded in the infinite plate, there is an open-end effect as discussed by Rokhlin. ${ }^{4}$ In spite of these discrepancies, the agreement shown here indicates that the sharp peaks and dips in Fig. 3 are brought about by the resonant interaction of the A0mode Lamb wave with the crack.

In the frequency range $0<f_{0} d / c_{\mathrm{T}}<0.2$, the magnitude of $F\left(x_{1}\right)$ in Eq. (31) is averaged over the crack and normalized to define the following parameter, which can be used as a measure of the opening displacement of the crack,

$$
\bar{F}=\frac{1}{2 A_{0}^{2} a} \int_{-a / 2}^{a / 2}\left\{\left(\operatorname{Re}\left[\bar{Y}_{2}\left(x_{1}\right)\right]\right)^{2}+\left(\operatorname{Im}\left[\bar{Y}_{2}\left(x_{1}\right)\right]\right)^{2}\right\} \mathrm{d} x_{1},
$$

(a)

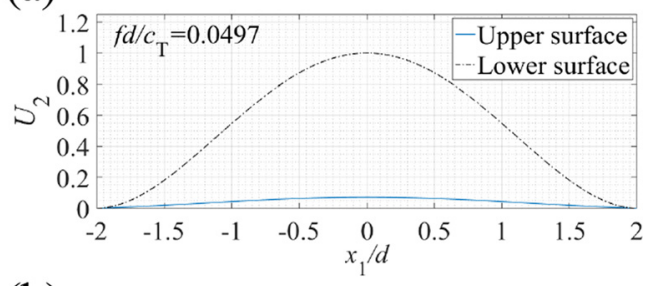

(b)

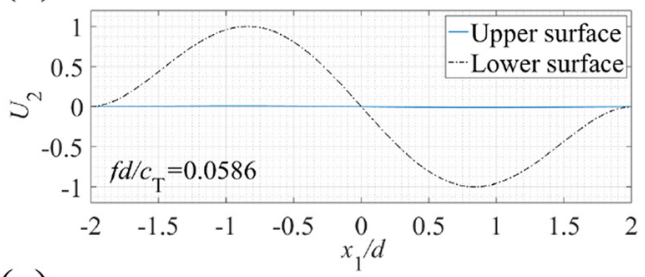

(c)

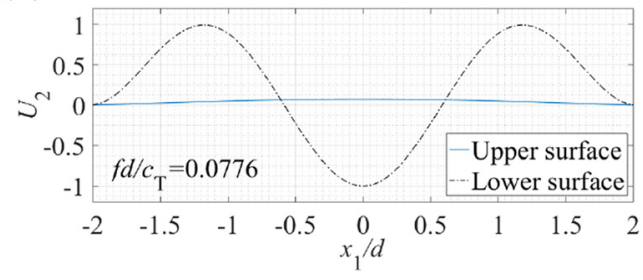

FIG. 6. (Color online) Three eigenmodes of the upper and lower surfaces of the thin layer for the eigenfrequencies of $f d / c_{\mathrm{T}}=$ (a) 0.0497, (b) 0.0586, and (c) 0.0776 when $a=4 d$. where $A_{0}$ is the aforementioned displacement amplitude of the incident A0 mode. This value is shown against $f_{0} d / c_{\mathrm{T}}$ in Fig. 7, which shows sharp peaks at the frequencies very close to the resonance frequencies found above. This indicates the dramatic increase of the relative displacements of crack surfaces by the resonance, which will give high driving force for the second-harmonic generation.

\section{B. Second-harmonic response}

For the reference case with $a=4 d$, the normalized magnitudes of the scattered second-harmonic A0 and S0 modes defined in Eq. (36) are shown in Fig. 8 against the normalized incident-wave frequency in the range $0<f_{0} d / c_{\mathrm{T}}$ $<0.1$. These magnitudes are shown in the logarithmic scale to examine their variation in a wider range. In Fig. 8, the back- and the forward-scattered second-harmonic A0 modes show sharp peaks at the incident-wave frequencies $f_{0} d / c_{\mathrm{T}}$ $=0.0268,0.0290,0.0375$, etc. The magnitudes of the scattered S0 modes also show peaks at these frequencies, but as a general trend, they are relatively small as compared to those of the scattered A0 modes.

By comparing the incident-wave frequencies which give sharp peaks (or dips) in the linear response and the peak frequencies in the second-harmonic response, one can find the following two simple relations between them. First, at the resonance frequencies in the linear response $\left(f_{0} d / c_{\mathrm{T}}\right.$ $=0.0536,0.0574,0.0754, \ldots$ in Fig. 3) or very close to them, the second-harmonic magnitudes also have peaks $\left(f_{0} d / c_{\mathrm{T}}=0.0527,0.0584,0.0754, \ldots\right.$ in Fig. 8$)$. Second, the second-harmonic magnitudes have peaks at some incidentwave frequencies $\left(f_{0} d / c_{\mathrm{T}}=0.0268,0.0290,0.0375, \ldots\right.$ in Fig. 8) when they are close to the half values of the linear resonance frequencies $\left(f_{0} d / c_{\mathrm{T}}=0.0536,0.0574,0.0754, \ldots\right.$ in Fig. 3). These two frequency selection rules can basically explain the location of all peaks in the second-harmonic response. The first rule can be interpreted based on the nature of the quadratic nonlinearity of the interface. Namely, the resonance induces larger relative normal

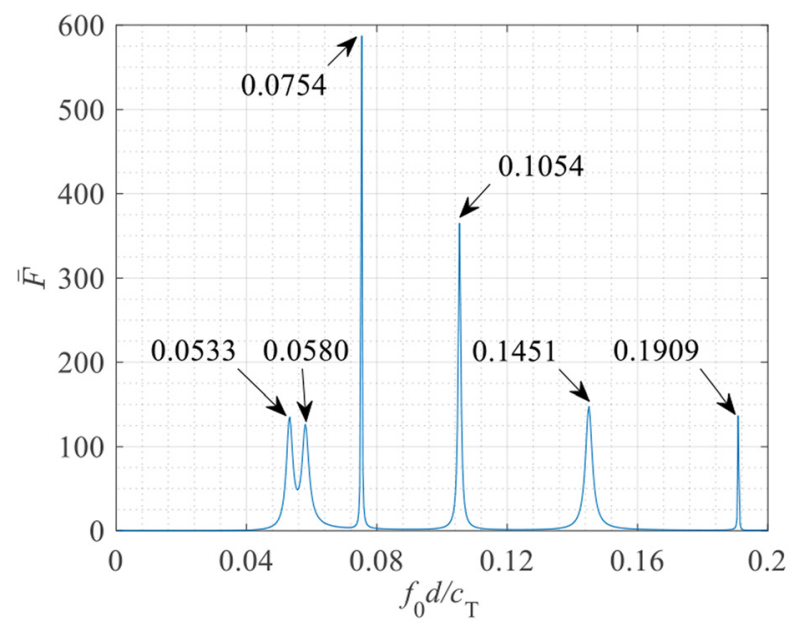

FIG. 7. (Color online) The crack opening parameter $\bar{F}$ against the normalized incident-wave frequency when $a=4 d$. 


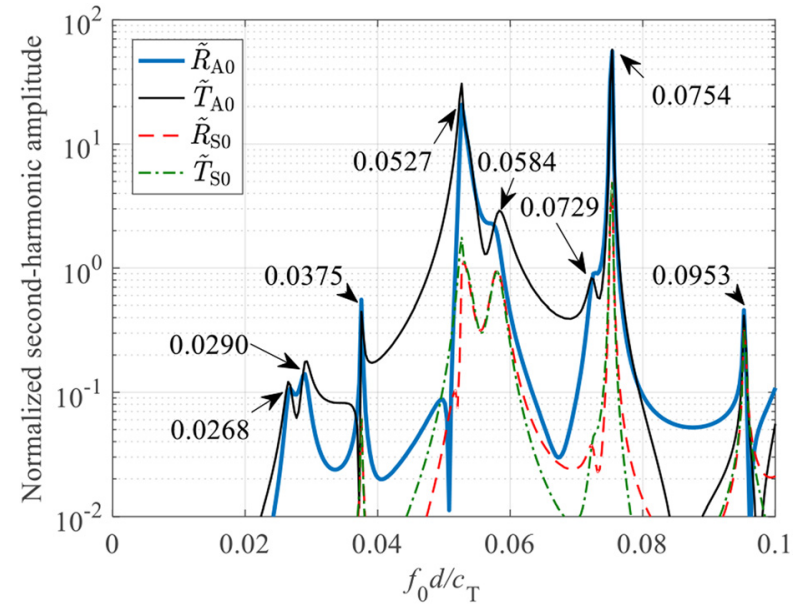

FIG. 8. (Color online) The second-harmonic amplitudes of the A0 and S0 modes against the normalized incident-wave frequency when $a=4 d$.

displacement of the crack (as shown in Fig. 7) and gives higher driving force for second-harmonic generation. The second rule can be interpreted as the enhancement of the nonlinear effect by the superharmonic resonance. Namely, the generated second-harmonic components can be amplified significantly when the double frequency matches the resonance of the crack. Some incident-wave frequencies $\left(f_{0} d / c_{\mathrm{T}}=0.0536(\fallingdotseq 0.0527), 0.0754\right)$ nearly meet both rules and are found to give particularly high peaks in the secondharmonic amplitudes. The results shown here thus support the idea of utilizing the so-called local defect resonance for efficient characterization of defects, as pioneered by Solodov and co-workers, ${ }^{38,39}$ and demonstrate its variant for Lamb waves.

It should be noted in passing that the quantities plotted in Fig. 8 are scaled by the non-dimensional parameter $\beta A_{0}$, as shown in Eq. (36). The actual magnitudes of the second-harmonic components, which depend on both these quantities and $\beta A_{0}$, can be significantly large at the peaks. The present analysis may lose accuracy for such high peaks

(a)

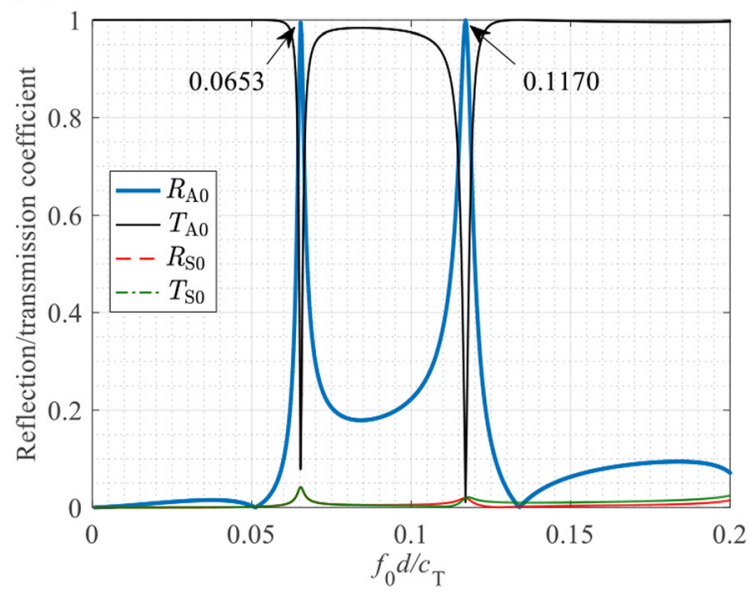

since it is based on the assumption of weak nonlinearity as stated in Sec. II. In fact, the precise magnitude of these peaks does not have much physical meaning since, in realistic circumstances, some damping mechanisms such as internal or interfacial friction can impede the occurrence of such high peaks. Nevertheless, the present analysis reveals clearly that the second-harmonic generation by a closed crack in a plate is highly frequency-sensitive due to the resonance of the crack to the incident Lamb wave.

In order to examine the effect of crack length on the linear and second-harmonic responses, the crack length is changed to $a=2 d$ and $6 d$ from the reference case $(a=4 d)$ with the other parameters kept the same. The numerical results are shown in Figs. 9 and 10. At a quick glance, the shorter crack in Fig. 9(a) has fewer resonances, while the longer crack in Fig. 10(a) has more resonances in the same frequency range as compared to the reference case $(a=4 d)$. This is naturally expected as the shorter (longer) crack should have higher (lower) resonance frequencies. In accordance with this, the second-harmonic magnitudes show a smaller (greater) number of peaks for the shorter (longer) crack. In Fig. 9(b) for the shorter crack $(a=2 d)$, the peaks of the second-harmonic amplitude are found at linear resonance $\left(f_{0} d / c_{\mathrm{T}}=0.0653\right)$ as well as near the halves of linear resonance $\left(f_{0} d / c_{\mathrm{T}}=0.0325,0.0584\right)$. In Fig. 10(b) for the longer crack $(a=6 d)$, the peaks are found at linear resonance $\left(f_{0} d / c_{\mathrm{T}}=0.0562,0.0659,0.0804\right)$ as well as near the halves of some linear resonance frequencies $\left(f_{0} d / c_{\mathrm{T}}\right.$ $=0.0262,0.0281,0.0331, \ldots)$. Therefore, the two frequency-selection rules discussed for the reference case are also valid for these cases.

\section{SUPPLEMENTARY ANALYSIS IN THE TIME DOMAIN}

The present analysis has revealed a significant effect of crack resonance on the linear and nonlinear scattering responses of the A0-mode Lamb wave. It is then of definite interest to examine the associated temporal reflection/

(b)

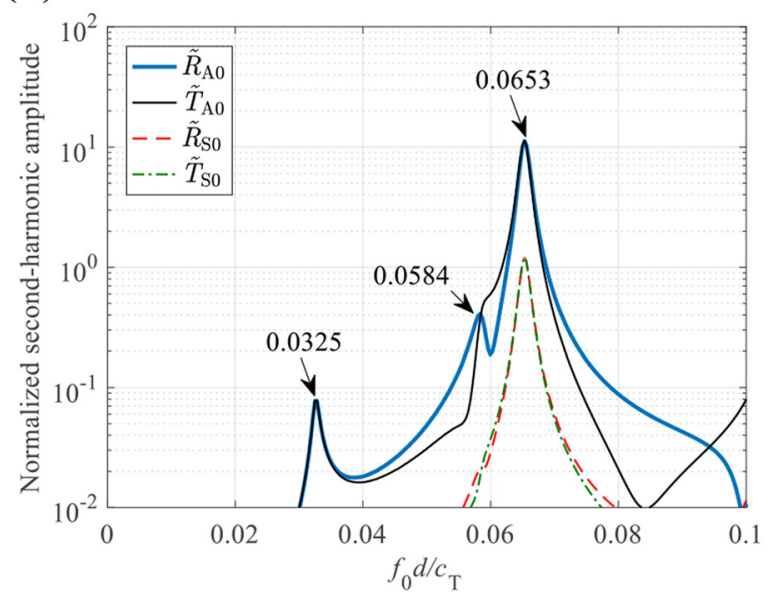

FIG. 9. (Color online) (a) The reflection and transmission coefficients and (b) the second-harmonic amplitudes of the A0 and S0 modes against the normalized incident-wave frequency when $a=2 d$. 
(a)

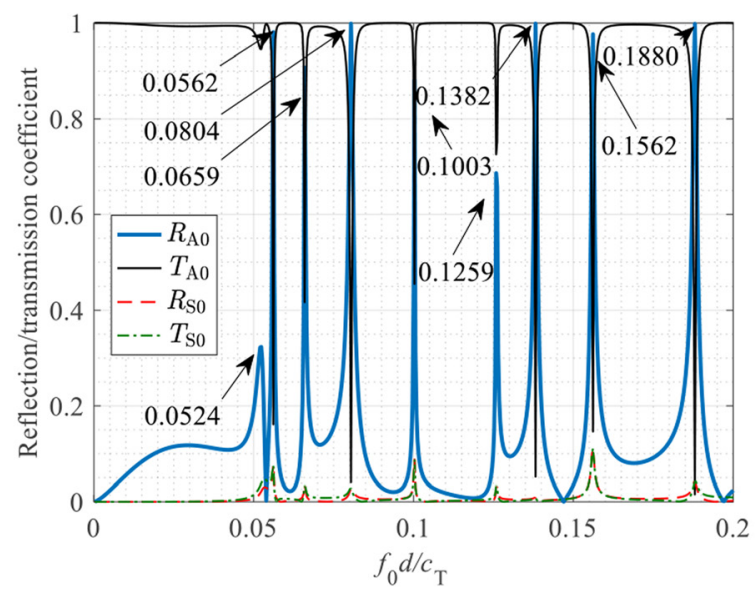

(b)

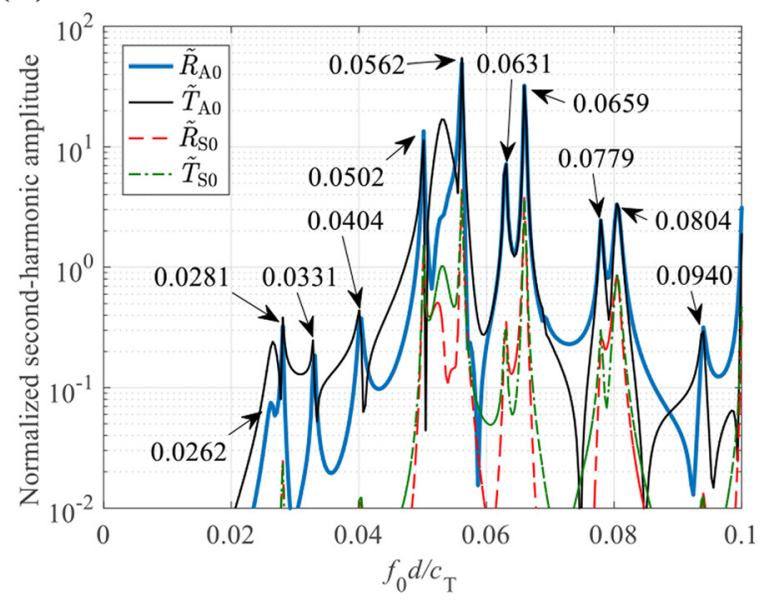

FIG. 10. (Color online) (a) The reflection and transmission coefficients and (b) the second-harmonic amplitudes of the A0 and S0 modes against the normalized incident-wave frequency when $a=6 d$.

transmission waveforms and their spectral components, since these are the features directly observed in actual measurements. To this purpose, the Lamb wave propagation in the plate with a closed crack is analyzed in the time domain using Comsol Multiphysics. The numerical model is constructed as shown in Fig. 11, for which the maximum element size is designed as $d / 20$ in accordance with the model used for the frequency-domain analysis. The wave source is set at $x_{1}=-55 d$ as the prescribed force pair with the magnitude as a function of time in the form of a sinusoidal wave of frequency $f_{0} d / c_{\mathrm{T}}=0.0757$ modulated by a Gaussian envelope. The selected center frequency of excitation is near one of the resonance frequencies of the crack (Fig. 3). The amplitude of the force is chosen so that it gives the maximum (the height of the wave-packet envelope) of the vertical displacement $A_{\mathrm{t} 0}=10^{-5} d$ at $x_{1}=x_{2}=0$ in the absence of the crack. This force pair excites the A0-mode Lamb wave in the plate and interacts with the crack. In order to suppress the unwanted reflection from the plate edges, the plate is connected to the exterior plates with the similar elastic constants but with the damping parameter that increases gradually with the distance from 0 to $E /\left(20 \pi f_{0}\right)$, where $E$ is Young's modulus. The time step for the analysis is set equal to $3.6 \times 10^{-4} / f_{0}$, which resolves the relevant period of wave motion with sufficient fineness and also meets the stability condition. Like in the frequency-domain analysis, the actual parameters are determined for aluminum alloy plates with $d=1 \mathrm{~mm}$.

The closed crack is modeled as a thin layer with the elastic constants equivalent to the spring-type interface as given in Eq. (32). In order to incorporate the quadratic nonlinearity of the crack, the thin layer is modeled as a nonlinear medium for which the shear modulus $\mu_{\mathrm{C}}$ is constant, but the bulk modulus $\kappa_{\mathrm{C}}$ varies linearly with the volume strain $\varepsilon_{\mathrm{v}}$ as

$$
\kappa_{\mathrm{C}}=\kappa_{\mathrm{C} 0}-\alpha \varepsilon_{\mathrm{V}}
$$

where $\kappa_{\mathrm{C} 0}$ and $\alpha$ are constants. The Nonlinear Structural Material module of Comsol Multiphysics is used to implement the above nonlinear material model. When the layer is sufficiently thin, this is approximately equivalent to the nonlinear spring-type interface model of Eqs. (1) and (2) with $\gamma=\chi=0$ when

$$
\kappa_{\mathrm{C} 0}=\left(K_{\mathrm{N}}-\frac{4}{3} K_{\mathrm{T}}\right) \delta, \mu_{\mathrm{C}}=K_{\mathrm{T}} \delta, \alpha=K_{\mathrm{N}} \delta^{2} \beta .
$$

Furthermore, this reduces to the thin layer model used in the frequency-domain analysis in Sec. IV when $\alpha=0$. In what follows, the reference case $\left(a=4 d, b=0.1 d, K_{\mathrm{N}} d /\left(\rho c_{\mathrm{T}}{ }^{2}\right)=\right.$ $\left.0.01, K_{\mathrm{T}} / K_{\mathrm{N}}=0.4\right)$ is considered and the nonlinearity parameter $\beta$ is chosen sufficiently small to ensure the weak nonlinearity, i.e., $\beta A_{\mathrm{t} 0}=0.005$. It is noted that this modeling, with a constant shear modulus and a volume-straindependent bulk modulus, is sufficient for the present analysis, but a more detailed account of nonlinear elastic thin layer modeling has been provided by Zhang et al. ${ }^{41}$ together with the discussion of its equivalence to the nonlinear spring-type interface model.

The vertical displacement histories at $x_{1}=-50 \mathrm{~d}, x_{2}=0$ (behind the crack) and $x_{1}=50 \mathrm{~d}, x_{2}=0$ (ahead of the crack), representing the reflected and transmitted A0-mode Lamb

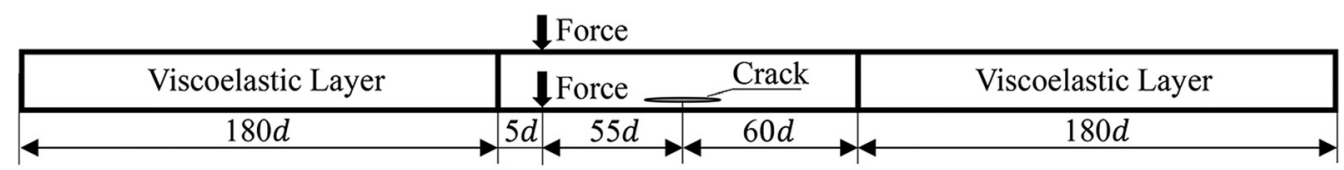

FIG. 11. The numerical model of the plate with a crack for the time-domain analysis. 
(a)

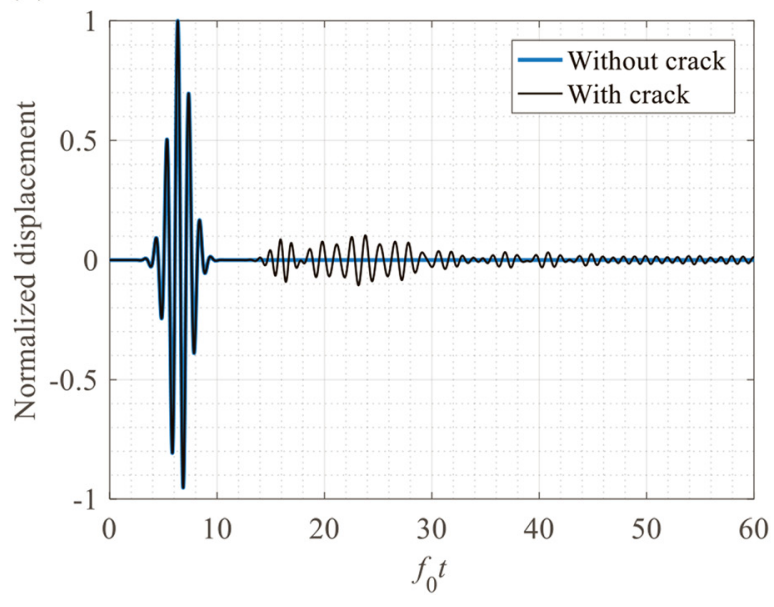

(b)

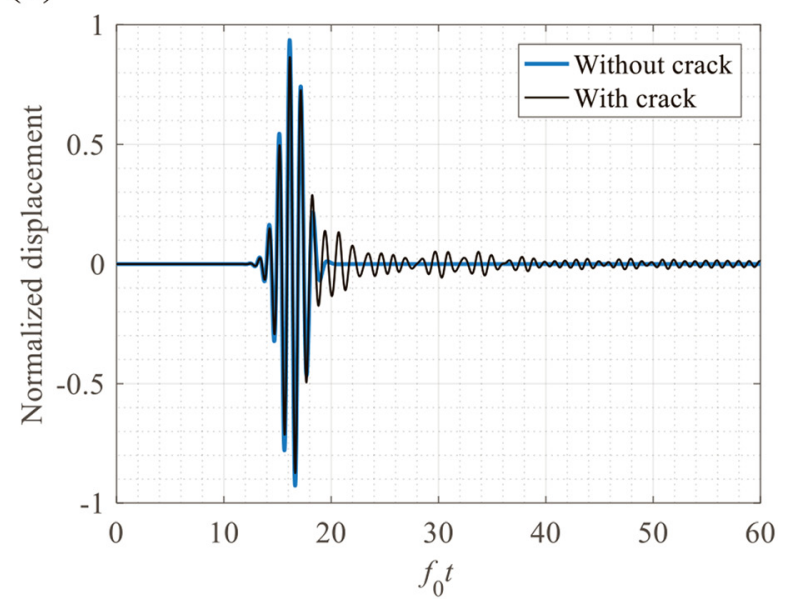

FIG. 12. (Color online) The time histories of the vertical displacement on the mid-plane at (a) $x_{1}=-50 d$ and (b) $x_{1}=50 d$ with and without the crack.

waves, are shown in Figs. 12(a) and 12(b) for the plates with and without a closed crack. The displacement is normalized by the maximum incident-wave amplitude $A_{\mathrm{t} 0}$, and the elapsed time is normalized as $f_{0} t$. At $x_{1}=-50 d$ [Fig. 12(a)], the first signal corresponds to the incident wave propagating to the right, which is followed by additional reflected wave signals in the presence of the crack. The reflected wave can be obtained as the difference of the two waveforms in Fig. 12(a). At $x_{1}=50 d$ [Fig. 12(b)], the wave consists of the direct transmission waveform and the accompanying oscillatory signals when the crack is present. The fast Fourier transforms of the reflected and transmitted waveforms are shown in Fig. 13 together with the amplitude spectrum of the incident wave obtained at $x_{1}=x_{2}=0$ in the plate without the crack. In Fig. 13, the amplitude spectra are normalized by the maximum of the incident wave spectrum. In the frequency bandwidth of the incident wave, the reflection and transmission coefficients $R_{\mathrm{t}}$ and $T_{\mathrm{t}}$ have been calculated by dividing the spectral amplitudes of reflected and transmitted waves by the corresponding amplitude of the incident

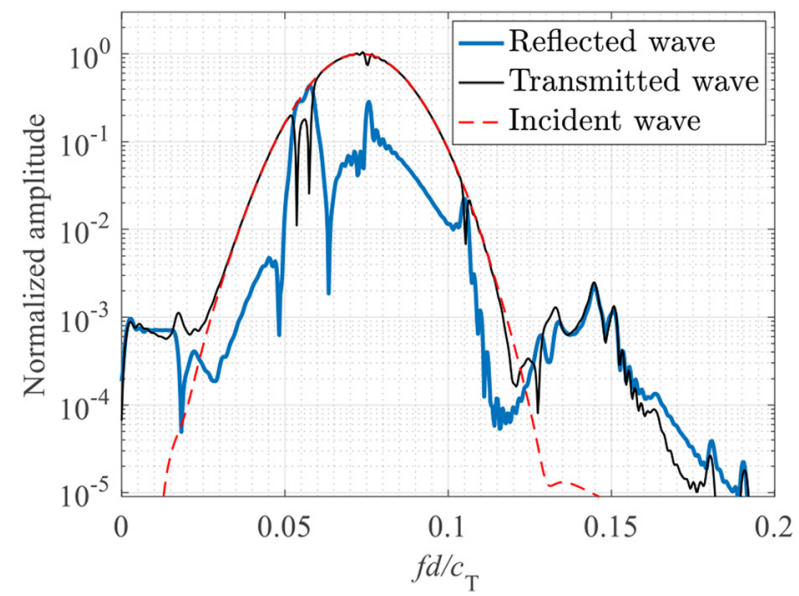

FIG. 13. (Color online) The amplitude spectra of the incident, reflected, and transmitted waves when $a=4 d$. wave, and excellent agreement with the results of the frequency-domain analysis in Sec. IV has been confirmed.

In Fig. 13, the reflected and transmitted waves have spectral amplitudes in the lower and higher frequencies outside the bandwidth of the incident wave. These spectral components are generated by the closed crack in the plate and can be interpreted as follows. The spectral components in the low-frequency region are likely due to the nonlinear interaction of amplitude-modulated waves observed for nonlinear media ${ }^{50,51}$ and have a common background as the static component due to the nonlinearity mentioned in Sec. II. The spectral components in the high-frequency range are also generated by the nonlinearity of the closed crack. They are shown separately in Fig. 14 and have peaks at some frequencies. The peak frequencies of the reflected A0-mode are indicated as texts in Fig. 14. The peaks at $f d / c_{\mathrm{T}}$ $=0.1448,0.1508$, and 0.1905 are likely the secondharmonic components of the incident wave component of the frequency $f d / c_{\mathrm{T}}=0.0724,0.0754$, and 0.0953, which are close or equal to the incident-wave frequencies of the second-harmonic peaks shown in Fig. 8. Namely, although the incident wave has a smooth Gaussian spectrum, some particular frequencies within its bandwidth generate the second-harmonic components in a selective manner due to the nonlinear interaction with the closed crack. This confirms the finding of the frequency-domain analysis obtained in Secs. II-IV in a qualitative manner. It should be noted that there are other peaks at the frequencies that do not correspond to the second-harmonics shown in Fig. 8, i.e., at $f d /$ $c_{\mathrm{T}}=0.1284,0.1337$, and 0.1807 . These are probably the mixed-frequency components that are also generated by the nonlinearity of the crack, since the sums of the resonance frequencies in Fig. $3\left(f_{0} d / c_{\mathrm{T}}=0.0536,0.0574,0.0754\right.$, and $0.1054)$ give the frequencies close to them, i.e., 0.0536 $+0.0754=0.129(\fallingdotseq 0.1284), 0.0574+0.0754=0.1328$ $(\fallingdotseq 0.1337), 0.0754+0.1054=0.1808(\fallingdotseq 0.1807)$. This indicates that some frequency components in the bandwidth of the incident wave are amplified by the crack resonance, and their combination frequencies are generated by the 


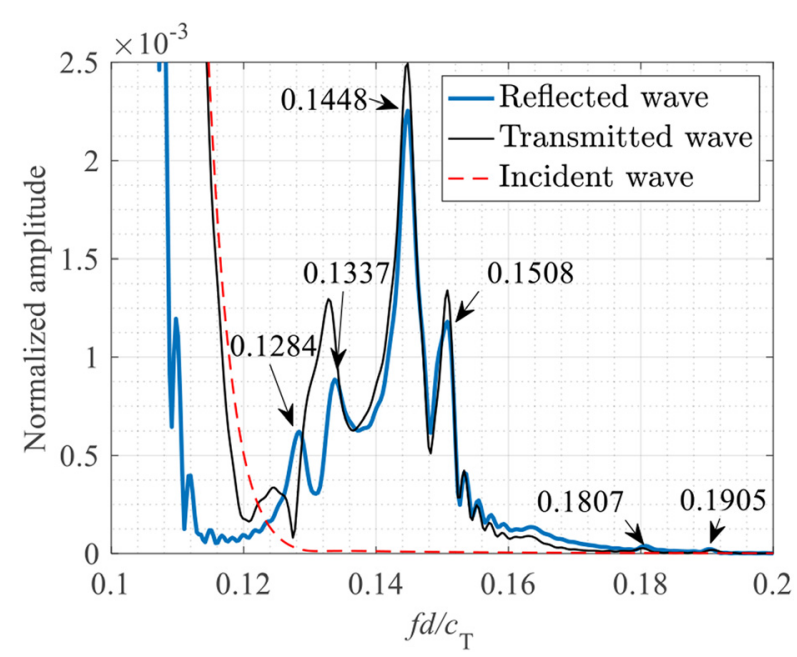

FIG. 14. (Color online) The amplitude spectra of the incident, reflected, and transmitted waves when $a=4 d$, magnified for the high-frequency region.

contact nonlinearity. Such mixed-frequency components are not obtained in the analysis of Secs. II-IV, which assumed the incidence of the Lamb mode with a single frequency. The analysis can, however, be extended to the case of the incidence of two waves with different frequencies in a straightforward manner.

The time-domain analysis presented here has shown that the reflected and transmitted Lamb waves contain specific nonlinear frequency components in a selective manner, even if the incident wave has a smooth spectrum. These frequency components may indicate the presence of a closed crack and contain information of its conditions. Understanding such features may be useful in interpreting the measurement data or selecting the suitable monitoring conditions for the Lamb-wave based nonlinear ultrasonic techniques.

\section{SUMMARY}

In this paper, the nonlinear interaction of the fundamental antisymmetric Lamb wave with a closed parallel crack in an elastic plate has been studied, with particular emphasis on the second-harmonic generation. Based on a perturbation method, the problem of nonlinear Lamb wave scattering by a closed crack has been decomposed into two linearized problems, i.e., for the linear reflection/transmission response and for the perturbation due to the weak nonlinearity of the crack, and solved by the finite element method in the frequency domain. Numerical results have shown significant effects of the resonance of the crack with the Lamb wave, which exhibits sharp peaks/dips in the reflection/transmission spectra and enhances the second-harmonic generation. The peak frequencies of the second-harmonic Lamb modes have been shown to depend on the size of the crack. Two simple frequency selection rules have been established that explain the enhancement of second-harmonic generation. The time-domain analysis has also been carried out to supplement the analysis, which has confirmed that the incident
Lamb wave interacts with the crack at specific frequencies within its bandwidth in a selective manner, as indicated in the present analysis. The perturbation method combined with the frequency-domain finite element analysis is an advantage in carrying out the computation of the reflection/ transmission and the associated second-harmonic generation characteristics of the Lamb wave with a given frequency in an efficient manner and elucidates the essential physics.

\section{ACKNOWLEDGMENTS}

This study has been supported by JSPS KAKENHI (No. JP19H02026). T.Y also acknowledges the financial support from the China Scholarship Council and the National Natural Science Foundation of China (Grant No. 11774264).

\section{APPENDIX}

In order to check the validity of replacing the springtype interface by a thin elastic layer, the guided wave propagation in a bilayer plate with a linear spring-type interface at $0.1 d$ above the lower surface as shown in Fig. 15(a) was examined. For the frequency-domain analysis with Comsol Multiphysics, the numerical model consisting of the elastic plate of the length $60 d$ connected with the PMLs of the length $30 d$ at both ends was built, where the spring-type interface was replaced by a thin elastic layer of thickness $\delta$ $=10^{-6} d$ as shown in Fig. 15(b). The elastic constants $\lambda_{\mathrm{C}}$ and $\mu_{\mathrm{C}}$ of the thin layer were determined by Eq. (32) for the two values of $K_{\mathrm{N}} d /\left(\rho c_{\mathrm{T}}^{2}\right)=0.01$ and 0.1 . The frequencydomain analysis was performed for a normal point force applied at the left edge of the plate region, and the horizontal displacement was extracted on the upper plate surface at 512 equally spaced points starting at the distance $5 d$ from the excitation point with the spatial sampling interval of $d / 10$ in the horizontal direction as shown in Fig. 15(b). The spatial fast Fourier transform (FFT) was applied to the sampling data computed at each excitation frequency in the range $0<f d / c_{\mathrm{T}}<0.6$ with interval of $\Delta f d / c_{\mathrm{T}}=3.155$ $\times 10^{-4}$, and the frequency-wavenumber plot was obtained as shown in Fig. 16 for $K_{\mathrm{N}} d /\left(\rho c_{\mathrm{T}}^{2}\right)=0.01$ and 0.1 with $K_{\mathrm{T}} /$ $K_{\mathrm{N}}=0.4$. In Fig. 16, the analytical dispersion curves of the corresponding bilayer plate with the spring-type interface are superposed, which were obtained by solving the global matrix of the system by introducing the linear spring-type

(a)

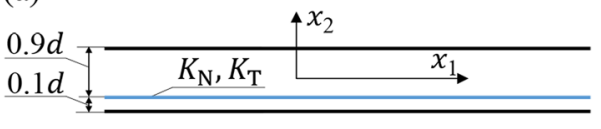

(b)

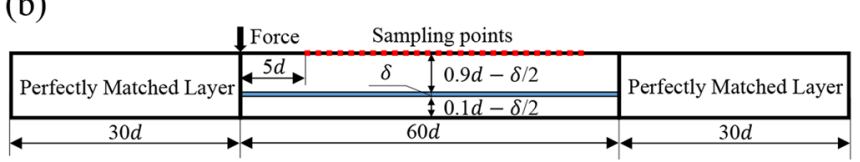

FIG. 15. (Color online) (a) A bilayer plate with a spring-type interface and (b) the numerical model of the plate with a thin elastic layer. 
(a)

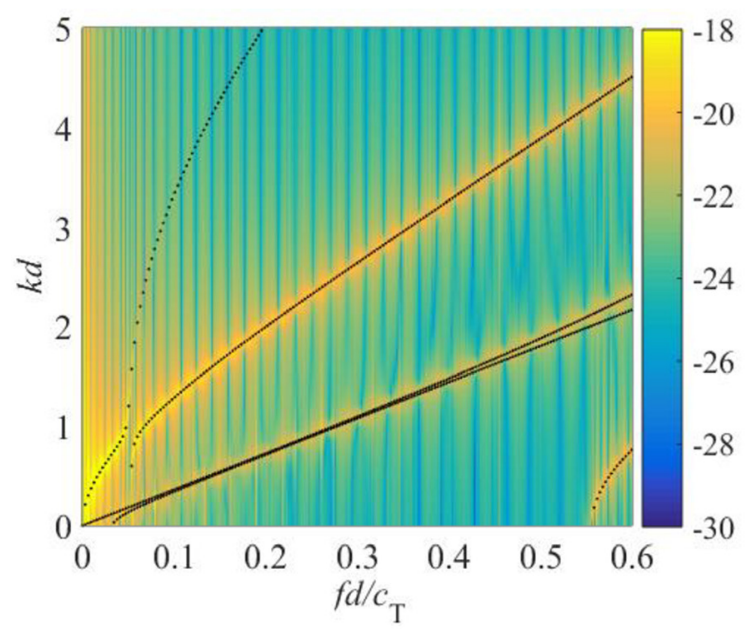

(b)

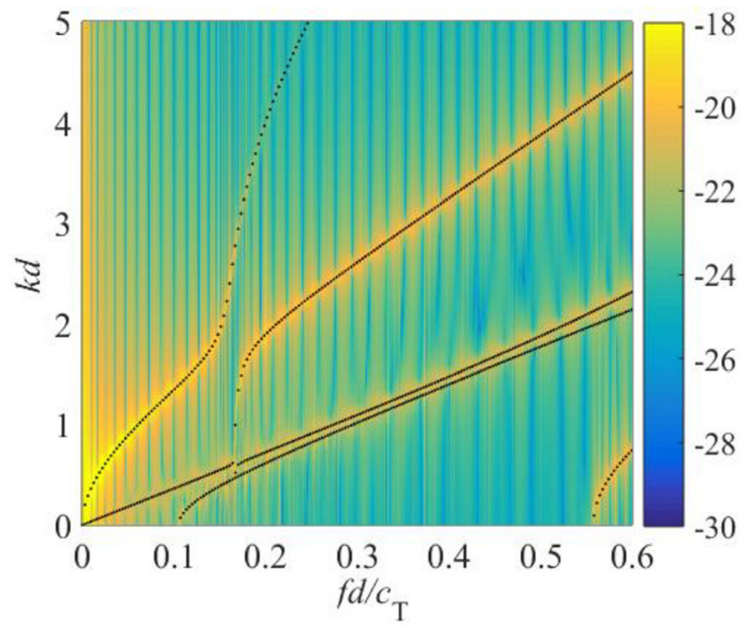

FIG. 16. (Color online) The frequency-wavenumber plots of the propagating wave in the plate with a thin elastic layer for $K_{\mathrm{N}} d /\left(\rho c_{\mathrm{T}}{ }^{2}\right)=(\mathrm{a}) 0.01$ and $(\mathrm{b}) 0.1$ together with the corresponding dispersion curves for the bilayer plate with a spring-type interface.

boundary conditions in Eqs. (16) and (17) in the procedure proposed by Lowe. ${ }^{52}$ It is shown in Fig. 16 that the results of the finite element analysis using the thin elastic layer match the analytical dispersion curves for the spring-type interface very well. Furthermore, the numerical values of the ratios of the stress and the relative displacement at the thin layer (i.e., $\left|\bar{\Sigma}_{22} / \bar{Y}_{2}\right|$ and $\left|\bar{\Sigma}_{12} / \bar{Y}_{1}\right|$ ) were checked by choosing arbitrary points on the upper and lower boundaries of the thin elastic layer. Theoretically, these values should coincide with the interfacial stiffnesses $K_{\mathrm{N}}$ and $K_{\mathrm{T}}$ for the linear spring-type interface. The numerical values by the finite element analysis assuming the thin layer were within negligible errors around the theoretical values, which also confirmed that the spring-type interface was accurately simulated by the thin elastic layer.

${ }^{1}$ I. A. Viktorov, Rayleigh and Lamb Waves, Physical Theory and Applications (Plenum Press, New York, 1967).

${ }^{2} \mathrm{P}$. Cawley and D. Alleyne, "The use of Lamb waves for the long range inspection of large structures," Ultrasonics 34, 287-290 (1996).

${ }^{3}$ S. Rokhlin, "Diffraction of Lamb waves by a finite crack in an elastic layer," J. Acoust. Soc. Am. 67, 1157-1165 (1980).

${ }^{4}$ S. I. Rokhlin, "Resonance phenomena of Lamb waves scattering by a finite crack in a solid layer," J. Acoust. Soc. Am. 69, 922-928 (1981).

${ }^{5}$ Y. Cho, D. D. Hongerholt, and J. L. Rose, "Lamb wave scattering analysis for reflector characterization," IEEE Trans. Ultrason. Ferroelectr. Freq. Control 44, 44-52 (1997).

${ }^{6}$ D. N. Alleyne and P. Cawley, "The interaction of Lamb waves with defects," IEEE Trans. Ultrason. Ferroelectr. Freq. Control 39, 381-397 (1992).

${ }^{7}$ M. Catstaings, E. Le Clezio, and B. Hosten, "Modal decomposition method for modeling the interaction of Lamb waves with cracks," J. Acoust. Soc. Am. 112, 2567-2582 (2002).

${ }^{8}$ M. J. S. Lowe and O. Diligent, "Low-frequency reflection characteristics of the s0 Lamb wave from a rectangular notch in a plate," J. Acoust. Soc. Am. 111, 64-74 (2002)

${ }^{9}$ M. J. S. Lowe, P. Cawley, J.-Y. Kao, and O. Diligent, “The low frequency reflection characteristics of the fundamental antisymmetric Lamb wave a0 from a rectangular notch in a plate," J. Acoust. Soc. Am. 112, 2612-2622 (2002).

${ }^{10}$ A. Gunawan and S. Hirose, "Mode-exciting method for Lamb wave scattering analysis," J. Acoust. Soc. Am. 115, 996-1005 (2004).
${ }^{11}$ M. A. Flores-López and R. D. Gregory, "Scattering of Rayleigh-Lamb waves by a surface breaking crack in an elastic plate," J. Acoust. Soc. Am. 119, 2041-2049 (2006).

${ }^{12}$ T. Kundu, A. Maji, T. Ghosh, and K. Maslov, "Detection of kissing bonds by Lamb waves," Ultrasonics 35, 573-580 (1998).

${ }^{13}$ B. C. Lee and W. J. Staszewski, "Modelling of Lamb wave interaction with open and closed fatigue cracks for damage detection," IOP Conf. Ser.: Mater. Sci. Eng. 10, 012059 (2010).

${ }^{14} \mathrm{H}$. Cho and C. J. Lissenden, "Structural health monitoring of fatigue crack growth in plate structures with ultrasonic guided waves," Struct. Health Monit. 11, 393-404 (2011).

${ }^{15}$ M. Matsushita, N. Mori, and S. Biwa, "Transmission of Lamb waves across a partially closed crack: Numerical analysis and experiment," Ultrasonics 92, 57-67 (2019).

${ }^{16}$ I. Y. Solodov, N. Krohn, and G. Busse, "CAN: An example of nonclassical acoustic nonlinearity in solids," Ultrasonics 40, 621-625 (2002).

${ }^{17}$ N. P. Yelve, M. Mitra, and P. M. Mujumdar, "Detection of stiffener disbonding in a stiffened aluminium panel using nonlinear Lamb wave," Appl. Acoust. 89, 267-272 (2015).

${ }^{18}$ N. P. Yelve, M. Mitra, P. M. Mujumdar, and C. Ramadas, "A hybrid method based upon nonlinear Lamb wave response for locating a delamination in composite laminates," Ultrasonics 70, 12-17 (2016).

${ }^{19}$ N. P. Yelve, M. Mitra, and P. M. Mujumdar, "Detection of delamination in composite laminates using Lamb wave based nonlinear method," Compos. Struct. 159, 257-266 (2017).

${ }^{20}$ R. Soleimanpour, C. T. Ng, and C. H. Wang, "Higher harmonic generation of guided waves at delaminations in laminated composite beams," Struct. Heal. Monit. 16, 400-417 (2017).

${ }^{21}$ X. Liu, L. Bo, K. Yang, Y. Liu, Y. Zhao, J. Zhang, N. Hu, and M. Deng, "Locating and imaging contact delamination based on chaotic detection of nonlinear Lamb waves," Mech. Syst. Signal Process. 109, 58-73 (2018).

${ }^{22} \mathrm{G}$. Shkerdin and C. Glorieux, "Nonlinear modulation of Lamb modes by clapping delamination," J. Acoust. Soc. Am. 124, 3397-3409 (2009).

${ }^{23} \mathrm{G}$. Shkerdin and C. Glorieux, "Nonlinear clapping modulation of lamb modes by normally closed delamination," IEEE Trans. Ultrason. Ferroelectr. Freq. Control 57, 1426-1433 (2010).

${ }^{24}$ S. Delrue and K. Van Den Abeele, "Three-dimensional finite element simulation of closed delaminations in composite materials," Ultrasonics 52, 315-324 (2012).

${ }^{25}$ S. Delrue and K. Van Den Abeele, "Detection of defect parameters using nonlinear air-coupled emission by ultrasonic guided waves at contact acoustic nonlinearities," Ultrasonics 63, 147-154 (2015).

${ }^{26}$ A. K. Singh, B. Y. Chen, V. B. C. Tan, T. E. Tay, and H. P. Lee, "Finite element modeling of nonlinear acoustics/ultrasonics for the detection of closed delaminations in composites," Ultrasonics 74, 89-98 (2017). 
${ }^{27} \mathrm{C}$. Pecorari, "Nonlinear interaction of plane ultrasonic waves with an interface between rough surfaces in contact," J. Acoust. Soc. Am. 113, 3065-3072 (2003).

${ }^{28}$ S. Biwa, S. Nakajima, and N. Ohno, "On the acoustic nonlinearity of solid-solid contact with pressure-dependent interface stiffness," Trans. ASME J. Appl. Mech. 71, 508-515 (2004).

${ }^{29}$ J.-Y. Kim and J.-S. Lee, "A micromechanical model for nonlinear acoustic properties of interfaces between solids," J. Appl. Phys. 101, 043501 (2007).

${ }^{30}$ S. Biwa, S. Hiraiwa, and E. Matsumoto, "Experimental and theoretical study of harmonic generation at contacting interface," Ultrasonics $\mathbf{4 4}$, e1319-e1322 (2006).

${ }^{31}$ J.-Y. Kim, A. Baltazar, J. W. Hu, and S. I. Rokhlin, "Hysteretic linear and nonlinear acoustic responses from pressed interfaces," Int. J. Solids Struct. 43, 6436-6452 (2006).

${ }^{32}$ S. Biwa, "Second-harmonic generation at contacting interfaces," in Nonlinear Ultrasonic and Vibro-Acoustical Techniques for Nondestructive Evaluation, edited by T. Kundu (Springer, Cham, Switzerland, 2019), pp. 263-299.

${ }^{33}$ N. Mori, S. Biwa, and T. Hayashi, "Reflection and transmission of Lamb waves at an imperfect joint of plates," J. Appl. Phys. 113, 074901 (2013).

${ }^{34} \mathrm{~N}$. Mori and S. Biwa, "Transmission of Lamb waves and resonance at an adhesive butt joint of plates," Ultrasonics 72, 80-88 (2016).

${ }^{35}$ N. Mori and S. Biwa, "Transmission characteristics of the S0 and A0 Lamb waves at contacting edges of plates," Ultrasonics 81, 93-99 (2017).

${ }^{36}$ N. Mori, S. Biwa, and T. Kusaka, "Harmonic generation a nonlinear imperfect joint of plates by the S0 Lamb wave incidence," Trans. ASME J. Appl. Mech. 86, 121003 (2019).

${ }^{37}$ S. Biwa and N. Ito, "Nonlinear response of a closed defect to Lamb waves: Perturbation analysis using hybrid finite element method," in Recent Advances in Structural Integrity Analysis-Proceedings of the International Congress (APCF/SIF-2014) (Woodhead Publishing, Sawston, UK, 2014) pp. 261-264.

${ }^{38}$ I. Solodov, J. Bai, S. Bekgulyan, and G. Busse, "A local defect resonance to enhance acoustic wave-defect interaction in ultrasonic nondestructive evaluation," Appl. Phys. Lett. 99, 211911 (2011).

${ }^{39}$ I. Solodov, "Resonant acoustic nonlinearity of defects for highly-efficient nonlinear NDE," J. Nondestr. Eval. 33, 252-262 (2014).
${ }^{40}$ Z. An, X. Wang, M. Deng, J. Mao, and M. Li, "A nonlinear spring model for an interface between two solids," Wave Motion 50, 295-309 (2013).

${ }^{41}$ Z. Zhang, P. Nagy, and W. Hassan, "Analytical and numerical modeling of non-collinear shear wave mixing at an imperfect interface," Ultrasonics 65, 165-176 (2016).

${ }^{42}$ Y. Ishii, S. Biwa, and T. Adachi, "Second-harmonic generation of twodimensional elastic wave propagation in an infinite layered structure with nonlinear spring-type interfaces," Wave Motion 96, 102569 (2020).

${ }^{43}$ J. L. Rose, Ultrasonic Waves in Solid Media (Cambridge University Press, Cambridge, 1999).

${ }^{44}$ P. B. Nagy, "Ultrasonic classification of imperfect interfaces," J. Nondestr. Eval. 11, 127-139 (1992).

${ }^{45}$ A. Baltazar, S. I. Rokhlin, and C. Pecorari, "On the relationship between ultrasonic and micromechanical properties of contacting rough surfaces," J. Mech. Phys. Solids 50, 1397-1416 (2002).

${ }^{46}$ D. Liaptsis, B. Drinkwater, and R. Thomas, "The interaction of oblique incidence ultrasound with rough, partially contacting interfaces," Nondestr. Test. Eval. 21, 109-121 (2006).

${ }^{47}$ S. Biwa, S. Hiraiwa, and E. Matsumoto, "Stiffness evaluation of contacting interfaces by bulk and interface waves," Ultrasonics 47, 123-129 (2007).

${ }^{48}$ S. Biwa, S. Hiraiwa, and E. Matsumoto, "Pressure-dependent stiffnesses and nonlinear ultrasonic response of contacting surfaces," J. Solid Mech. Mater. Eng. 3, 10-21 (2009).

${ }^{49}$ S. I. Rokhlin and Y. J. Wang, "Analysis of boundary conditions for elastic wave interaction with an interface between two solids," J. Acoust. Soc. Am. 89, 503-515 (1991).

${ }^{50}$ B. K. Novikov and O. V. Rudenko, "Generation of low-frequency harmonics in the field of a high-power amplitude-modulated wave," Sov. Phys. Acoust. 23, 455-459 (1977).

${ }^{51}$ S. Biwa, K. Nagae, C. Inserra, and E. Matsumoto, "Evaluation of nonlinear low-frequency components generated by amplitude-modulated waves in a carbon/carbon composite," AIP Conf. Proc. 1433, 497-500 (2012).

${ }^{52}$ M. J. S. Lowe, "Matrix techniques for modeling ultrasonic waves in multilayered media," IEEE Trans. Ultrason. Ferroelectr. Freq. Control 42, 525-542 (1995). 